\title{
Miszellen
}

Michael Lurie

\section{Der schiffbrüchige Odysseus oder: Wie Arkesilaos zum Skeptiker wurde}

\author{
Zu Timon von Phleius Fr. 806 SH (32 D)
}

Keywords: Timon von Phleius, Pyrrhon, Skeptizismus, Arkesilaos, Odyssee

DOI 10.1515/phil-2014-0011

Den Sinn der Silloi, der Philosophensatire des Timon von Phleius, machen bekanntlich vor allem zwei Elemente aus. Zum einen ist es der unmittelbare, nicht selten burleske Spott, der sich gegen einzelne, in erster Linie dogmatische Philosophen richtet. Zum anderen stellt Timon diesem philosophischen Spott auch Homer-Parodie in den Dienst. Die häufigen, durch Zitate von Versen oder auch nur einzelnen Versteilen und Wörtern markierten Anspielungen auf die Ilias und Odyssee sind oft implikationsreich, weil sie den Leser dazu einladen, die Figuren und Situationen der Satire jeweils mit bestimmten Gestalten, Szenen und Situationen der homerischen Epen zu vergleichen, und dadurch dem Spott und der Komik eine zusätzliche Dimension verleihen' ${ }^{1}$.

1 Generell zu Timon, seinen philosophischen Positionen und seiner Philosophensatire, siehe W. Görler, Älterer Pyrrhonismus. Jüngere Akademie. Antiochos von Ascalon, in: H. Flashar (Hrsg.), Grundriß der Geschichte der Philosophie. Die Philosophie der Antike, Bd. 4: Die Hellenistische Philosophie, Basel 1994, 719-989, hier 760-767 mit älterer Literatur und weiterführenden Hinweisen und jetzt auch D. L. Clayman, Timon of Phlius: Pyrrhonism Into Poetry, Berlin 2009. Die oft vernachlässigte Bedeutung der Homer-Parodie als eines wichtigen Schlüssels zum Verständnis von Timons Silloi hat W. Ax, Timons Gang in die Unterwelt, Hermes 119, 1991, 177193 (wieder abgedruckt in: ders., Text und Stil. Studien zur antiken Literatur und deren Rezeption, hrsg. von C. Schwarz, Stuttgart 2006, 9-25) mit Nachdruck hervorgehoben. Verschiedene Aspekte und Funktionen der Homer-Parodie in griechischer Literatur sind jetzt in B. Acosta-Hughes et al. (eds.), Homère revisité: parodie et humour dans les réécritures homériques, Besançon 2011 behandelt.

Michael Lurie: National Humanities Center, 7 Alexander Drive, P. O. Box 12256, Research Triangle Park, North Carolina 27709-2256, USA, E-Mail: mlurie09@gmail.com 
Dass man dem gegen Arkesilaos, den Begründer der skeptischen Akademie, gerichteten Fragment SH 806 (32D) bis jetzt herzlich wenig Sinn und Komik abgewinnen konnte, hängt nun damit zusammen, dass keines der beiden komischen Sinn-Elemente ermittelt werden konnte. Fest stand - dank D. L. 4, 33 p. 281, 4282, 1 (Marcovich) - lediglich, dass das Fragment, zusammen mit dem zu derselben Szene gehörenden Fr. 805 SH (31D), einen Angriff auf Arkesilaos als einen opportunistischen, eklektischen und höchst unoriginellen Denker enthielt: in Wirklichkeit verdanke Arkesilaos seine skeptischen Anschauungen dem maßgeblichen Einfluss des einzig wahren Skeptikers - und Timons Lehrers - Pyrrhon, und seine Philosophie sei im Wesentlichen ein aus pyrrhonischer Skepsis und megarischer Dialektik zusammengeflicktes Machwerk ${ }^{2}$. Doch was konkret an diesem Fragment lustig sein soll und in welcher Situation sich Arkesilaos in Timons Silloi befindet, ob er hier als Schiff oder als Schwimmvogel, ${ }^{3}$ als Fisch, ${ }^{4}$ oder aber - was allerdings nicht viel plausibler, dafür aber viel weniger lustig wäre - als ein Athlet bei Gewichtsübungen ${ }^{5}$ dargestellt war, musste mangels

2 Zur Frage des Einflusses der pyrrhonischen Skepsis und der megarischen Schule auf Arkesilaos sowie zu dem historischen und philosophischen Kern der von Timon hier spielerisch behandelten Entwicklungen siehe Görler (1994) 812-816 mit weiterführenden Hinweisen sowie D. Sedley, The motivation of Greek scepticism', in: M. F. Burnyeat (ed.), The Skeptical Tradition, Berkeley/Los Angeles 1983, 9-29; F. Decleva Caizzi, Pirroniani ed Accademici nel III secolo a. c., in: H. Flashar und O. Gigon (Hgg.), Aspects de la philosophie Hellénistique (= Entretiens sur l'antiquité classique 32), Genêve 1986, 147-83; A. A. Long/D. Sedley (eds.), The Hellenistic Philosophers, vol. 1-2, Cambridge 1987, hier 1. 445-448; J. Brunschwig, Once again on Eusebius on Aristocles on Timon on Pyrrho, in: ders. (Hrsg.), Papers in Hellenistic Philosophy, Cambridge 1994, 190-211, hier 206f.; M. Frede, The Sceptics, in: Routledge History of Philosophy, vol. II: From Aristotle to Augustine, ed. by D. Furley, London/New York 1999, 253-286 und M. Schofield, Academic Epistemology, in: Cambridge History of Hellenistic Philosophy, ed. by K. Algra/J. Barnes/J. Mansfeld/M. Schofield, Cambridge 1999, 323-351, bes. $330 \mathrm{f}$.

3 Beides wurde von U. von Wilamowitz-Moellendorff, Antigonos von Karystos, Berlin 1881, 72 vermutet, ablehnend C. Wachsmuth, Corpusculum Poesis. Epicae Graecae Ludibundae, Fasc. 2: Sillographorum Graecorum Reliquae, Leipzig ${ }^{2} 1885$, 116-118, dessen eigener Vorschlag - eine Schlachtszene zwischen Arkesilaos und Zeno - freilich ebenso wenig überzeugend scheint.

4 H. Diels, Poetarum Philosophorum Fragmenta, Berlin 1901, 183 und 192 ad loc.; leicht modifiziert in A. A. Long, Timon of Phlius: Pyrrhonist and Satirist, PCPhS 204, N. S. 24, 1978, 68-91 (wieder abgedruckt in: ders., From Epicurus to Epictetus, Oxford 2006, 90-95) 79f. Die einst einflussreiche Deutung wurde von M. Billerbeck, Faule Fische. Zu Timon von Phleius und seiner Philosophensatire, MH 44, 1987, 128-133; M. di Marco, Timone di Fliunte: Silli. Introduzione, edizione critica, traduzione e commento, Rome 1989, 182-186, und jetzt auch Clayman (2009) 107-112 erneut in Frage gestellt; vgl. dagegen Ax (1991) 14 mit Anm. 18 und R. F. Glei, Rezension von Di Marco (1989), Gnomon 65, 1993, 112-115, bes. 112f. 115.

5 Billerbeck (1987) 130-132, zustimmend Görler (1994) 812, ablehnend Ax (1991) 182f. mit Anm. 18; vgl. Di Marco (1989) 184 f. 
fester Anhaltspunkte der spekulativen Einbildungskraft einzelner Interpreten überlassen bleiben. Aber auch als Homer-Parodie schien das rätselhafte Fragment unergiebig. Während Timon in dem deshalb auch viel mehr, wenn auch nicht mit viel mehr Erfolg, besprochenen Fr. 805 SH (31 D) immerhin Od. 5, 346 im Sinne zu haben schien, glaubte man für das Fr. 806 SH (32 D) nicht einmal eine Homer-Vorlage finden zu können.

Indes besteht kein Zweifel, dass Timon in Fr. $806 \mathrm{SH}$ :

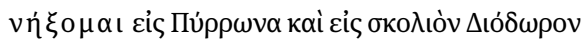

auf dieselbe Szene im 5. Buch der Odyssee, und zwar auf Od. 5, 364, auf den letzten Vers der verzweifelten Rede des schiffbrüchigen, gegen den Sturm kämpfenden Odysseus also, anspielt:

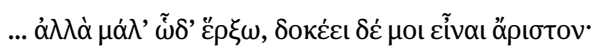

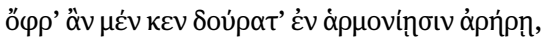

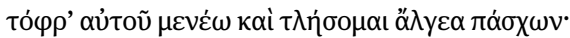

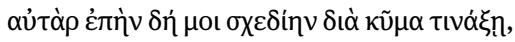

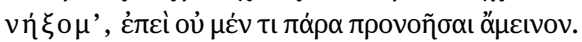

Die bislang nicht beachtete Anspielung scheint für das Verständnis der Komik des Fragments und wohl auch der ganzen Szene, der es zusammen mit Fr. 805 SH (31D) ursprünglich entstammt, von entscheidender Bedeutung zu sein ${ }^{6}$. Es ist nämlich gewiss kein Zufall, dass Timon in den Versen, in denen er sich über den opportunistischen Eklektizismus des wendigen Arkesilaos mokierte, indem er ihn vor lauter Ratlosigkeit zum Skeptiker Pyrrhon und Dialektiker Diodor schwimmen ließ, gleich zweimal auf die Szene aus dem 5. Buch der Odyssee anspielte, in der der schiffbrüchige, dem Gewitter schutzlos ausgelieferte, rat- und orientierungslose, jedoch nach wie vor wendige, energisch ums Überleben kämpfende Odysseus einfach dahinzuschwimmen beschließt, weil ihm nichts Besseres einfällt.

6 Wachsmuth ('1885) 117f.; Diels (1901) 192; H. Lloyd-Jones/P. Parsons (edd.), Supplementum Hellenisticum, Berlin/New York 1983, 379f. und di Marco, Timone di Fliunte: Silli. Introduzione, edizione critica, traduzione e commento, Rome 1989 ad loc. (fr. 32) gaben für diesen Vers keine Homervorlage an. Nur Billerbeck (1987) 131 Anm. 18 erwog beiläufig Od. 5, 364, ohne jedoch ihre

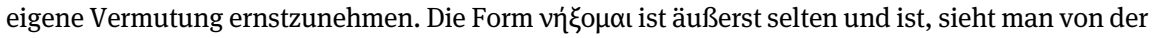
ähnlichen Anspielung auf dieselbe Odyssee-Stelle in Musaios 208 (ebenso am Versanfang) ab, sonst nirgends belegt. Da Fr. 805 SH (31D) auf dieselbe Episode anspielt, liegt die Vermutung nahe, dass Timon hier nicht einzelne Odyssee-Verse, sondern die ganze Szene im 5. Buch der Odyseee parodierte. Die Anspielung auf Od. 5, 346 in Fr. 805 SH hat erst Clayman (2009) 109f. auszuwerten versucht. 
Bedenkt man die von Timon zweifellos intendierte Anspielung auf die verzweifelte Situation und Worte des schiffbrüchigen Odysseus, so bekommen die Situation und die Worte des ratlosen, philosophisch desorientierten Arkesilaos in Fr. 806 SH erst überhaupt einen Sinn. Sie implizieren auf eine humorvolle Art und Weise den Vorwurf, dass Arkesilaos, von Hause aus ein Dogmatiker, erst in höchster Not, als sein dogmatisches Schiff in die Brüche ging, nicht aus Überzeugung, sondern um des reinen Überlebens willen zum wahren Skeptiker Pyrrhon und dem Dialektiker Diodor philosophische Zuflucht zu nehmen beschlossen habe. So erweist sich der Begründer der skeptischen Akademie als ein schlauer, skrupelloser Wendehals - als der schiffbrüchige Odysseus der griechischen Philosophiegeschichte ${ }^{7}$. Als Homer-Parodie verstanden, vermag vielleicht das spöttische Fragment den Leser auch heute noch zum Lachen oder zumindest zum Schmunzeln zu bringen.

Danksagung: Wolfram Ax, Hans Bernsdorff, Thomas Maißen und Marek Wecowski danke ich für wertvolle Hinweise und Anregungen.

7 Zur Rezeption der Gestalt des Odysseus in der griechischen Philosophie siehe jetzt S. Montiglio, From Villain to Hero. Odysseus in Ancient Thought, Ann Arbor 2011, die freilich auf Timons Silloi nicht eingeht. 